UPR1130-T

\title{
The Dynamics of Small Instanton Phase Transitions
}

\author{
Alexander Borisov ${ }^{1}$, Evgeny I. Buchbinder ${ }^{2}$, Burt A. Ovrut ${ }^{1}$ * \\ ${ }^{1}$ Department of Physics and Astronomy, University of Pennsylvania, \\ Philadelphia, PA 19104 \\ ${ }^{2}$ School of Natural Sciences, Institute for Advanced Study \\ Einstein Drive, Princeton, NJ 08540
}

\begin{abstract}
The small instanton transition of a five-brane colliding with one end of the $\mathbf{S}^{1} / \mathbf{Z}_{2}$ interval in heterotic $M$-theory is discussed, with emphasis on the transition moduli, their potential function and the associated non-perturbative superpotential. Using numerical methods, the equations of motion of these moduli coupled to an expanding Friedmann-Robertson-Walker spacetime are solved including nonperturbative interactions. It is shown that the five-brane collides with the end of the interval at a small instanton. However, the moduli then continue to evolve to an isolated minimum of the potential, where they are trapped by gravitational damping. The torsion free sheaf at the small instanton is "smoothed out" into a vector bundle at the isolated minimum, thus dynamically completing the small instanton phase transition. Radiative damping at the origin of moduli space is discussed and shown to be insufficient to trap the moduli at the small instanton point.
\end{abstract}

*borisov@sas.upenn.edu; evgeny@ias.edu; ovrut@physics.upenn.edu 


\section{Introduction:}

In [1, Witten studied $S O(32)$ heterotic string theory on $\mathbf{R}^{6} \times \mathbf{K} \mathbf{3}$ with an $S O(N), 4 \leq N \leq 32$ gauge instanton supported on $\mathbf{K 3}$ with instanton number 24 . The effective $\mathbf{R}^{6}$ theory manifests $N=2$ supersymmetry and has gauge symmetry $S O(32-N)$. The boundary of instanton moduli space contains singular points, at each of which one unit of instanton number is concentrated. These are called "small" instantons and the local moduli space $\mathcal{M}^{\prime}$ was shown to be $4 N-12$ dimensional. It was demonstrated in [1] that $\mathcal{M}^{\prime}$ can be described as the flat directions of a potential energy function. This is constructed from $N$ hypermultiplets, each transforming as a fundamental $N$ representation of $S O(N)$ and as a doublet under an additional $S U(2)$ gauge symmetry. At the origin of field space, all of these fields become massless and the $S U(2)$ symmetry becomes exact. Away from this point, however, the $S U(2)$ symmetry is spontaneously broken and only the $4 N-12$ moduli of $\mathcal{M}^{\prime}$ remain massless. One concludes that as one approaches a small instanton point in moduli space, the theory develops extra (massless) species and an enhanced symmetry. Such points are often referred to as being ESP.

Small instantons also occur in $M$-theory on $\mathbf{R}^{4} \times X \times \mathbf{S}^{1} / \mathbf{Z}_{2}$, where $X$ is a Calabi-Yau threefold [2, 3]. As first discussed in [2], to be consistent these vacua must have, supported on the Calabi-Yau manifold at each end of the $\mathbf{S}^{1} / \mathbf{Z}_{2}$ interval, a gauge instanton indexed by a subgroup of $E_{8}$. One can, for simplicity, assume a trivial instanton at one end of the interval. The non-trivial instanton on the other end is a connection on a holomorphic vector bundle $V$ over $X$ with structure group $G \subset E_{8}$. Quantum consistency requires that the instanton number, here generalized to the 2nd Chern class $c_{2}(V)$, must satisfy the constraint $c_{2}(V)=c_{2}(T X)-\mathcal{W}$, where $T X$ is the tangent bundle of the Calabi-Yau threefold and $\mathcal{W}$ is an effective class corresponding to M-five-branes in the $\mathbf{S}^{1} / \mathbf{Z}_{2}$ "bulk" space. The effective $\mathbf{R}^{4}$ theory manifests $N=1$ supersymmetry. Its gauge symmetry depends on the choice of structure group $G$. In a series of publications [4, 5], holomorphic vector bundles with $G=S U(N)$ were constructed. We will refer to these when specificity is required. For example, when $G=S U(3), S U(4)$ and $S U(5)$, the effective $\mathbf{R}^{4}$ gauge symmetry is $E_{6}, \operatorname{Spin}(10)$ and $S U(5)$ respectively.

The boundary of instanton moduli space contains singular points where a portion of the vector bundle becomes concentrated as a torsion free sheaf over an isolated holomorphic curve with effective class $\mathcal{C}^{\prime}$. These are the heterotic $M$-theory analogs of small instantons. Here, however, the local 
moduli spaces $\mathcal{M}^{\prime}$ are more complicated, changing with the structure of the Calabi-Yau threefold, the holomorphic vector bundle and the choice of $\mathcal{C}^{\prime}$. The moduli spaces of small instantons on elliptic Calabi-Yau threefolds over Hirzebruch bases $B=\mathbf{F}_{r}$ with vector bundles constructed from spectral covers were analyzed in several publications [6]. For example, consider $B=\mathbf{F}_{1}$, $G=S U(3)$ and $\mathcal{C}^{\prime}=\mathcal{S}$, with $\mathcal{S}$ a section of $\mathbf{F}_{1}$. Then, it was shown that $\operatorname{dim} \mathcal{M}^{\prime}=3(b-a)-6$, where $a, b$ are integers satisfying the constraints $a>6$ and $b>a+3$. It is expected that any $\mathcal{M}^{\prime}$ can again be described as the flat directions of a potential energy function. This would be constructed from chiral supermultiplets transforming in some representation $R$ of $G$ and, possibly, as a representation $r$ of an additional gauge symmetry $H$. At the origin of field space, all of these fields become massless and the $H$ symmetry becomes exact. Away from this point, the $H$ symmetry is spontaneously broken and only the moduli of $\mathcal{M}^{\prime}$ remain massless. Therefore, as one approaches a small instanton in moduli space, the theory develops extra (massless) species and, possibly, an enhanced symmetry. That is, small instantons in heterotic $M$-theory are $E S P$. Unfortunately, such potential functions have not been explicitly constructed for most moduli spaces. Limited simple examples indicate that such potentials exist, but a general proof is still lacking. Be that as it may, in this paper we will assume that small instanton moduli spaces $\mathcal{M}^{\prime}$ in heterotic $M$-theory can be so described.

In heterotic $M$-theory, strings wrapping a holomorphic curve in the CalabiYau threefold will generate a non-perturbative superpotential for both geometric and vector bundle moduli [7, 8]. As first discussed in [9], the superpotential created by a such a "worldsheet instanton" has a specific form; namely, an exponential of geometric and curve moduli multiplied by a "Pfaffian". The Pfaffian is a function of the determinant of the Dirac operator twisted by the vector bundle restricted to the curve. It is generically true [10] that the Pfaffian evaluated at any holomorphic curve is a homogeneous polynomial of the moduli of the restricted bundle. This has important implications for small instantons. It was shown in [10, 11] that the moduli of the vector bundle restricted to the small instanton curve $\mathcal{C}^{\prime}$ are precisely those which parameterize $\mathcal{M}^{\prime}$. Hence, strings wrapped on $\mathcal{C}^{\prime}$ generate a superpotential on $\mathcal{M}^{\prime}$ which "lifts" some, or all, of the flat directions. Furthermore, new local minima may now occur substantially away from the ESP at the zero of field space. Within the context of $B=\mathbf{F}_{r}, G=S U(3)$ and $\mathcal{C}^{\prime}=\mathcal{S}$, Pfaffians were explicitly computed in [10, 11]. For example, for $r=1, a>5$ and $b=a+6, \operatorname{dim} \mathcal{M}^{\prime}=3(6)-6=12$ and the Pfaffian was shown to be an 
explicit homogeneous polynomial of degree 5 in 7 of the 12 moduli of $\mathcal{M}^{\prime}$.

At a small instanton in heterotic $M$-theory, the vector bundle $V$ degenerates into the "union" of a new holomorphic vector bundle $V^{\prime}$ over $X$ and a sheaf over the curve $\mathcal{C}^{\prime}$. Because of the existence of the $\mathbf{S}^{1} / \mathbf{Z}_{2}$ interval, the sheaf can "detach" from $X$, leaving only $V^{\prime}$ behind, and become a component of a new M-five-brane curve in the bulk space. That is, $\mathcal{C}^{\prime} \subset \mathcal{W}^{\prime}$. The physical interpretation of this is that the small instanton "emits" a five-brane into the bulk space. Of course, this can occur in reverse, a component $\mathcal{C}^{\prime} \subset \mathcal{W}^{\prime}$ of a bulk space five-brane being "absorbed" into $V^{\prime}$ via a small instanton to become $V$. Such a process, either absorbing or emitting a bulk five-brane, is called a small instanton phase transition and was defined and studied in 12 . It was shown that there are two types of small instanton transitions, the first in which the structure group of the vector bundle is unchanged while the number of chiral zero modes is altered and a second where the reverse is true, the structure group changes while the number of chiral zero modes remains fixed. For an elliptically fibered Calabi-Yau threefold $X$, the first type of transition occurs when $\mathcal{C}^{\prime}$ is the pull-back of a curve in the base $B$. This is analogous to the heterotic $S O(32)$ case. The second type of transition takes place when $\mathcal{C}^{\prime}$ is proportional to the elliptic fiber $F$. In this paper, we will consider only those small instanton transition for which the structure group is unchanged.

An important aspect of any small instanton transition is that the $M$ five-brane wrapped on $\mathcal{C}^{\prime}$ in the bulk space itself has moduli. The most conspicuous of these is the translation modulus, the real part of which describes the separation of the five-brane from the end of the $\mathbf{S}^{1} / \mathbf{Z}_{2}$ interval and whose imaginary part is an axion. For a Calabi-Yau threefold $X$ elliptically fibered over any base $B$, the complete moduli space $\mathcal{M}_{\mathcal{C}^{\prime}}$ of an $M$-five-brane wrapped on $\mathcal{C}^{\prime}$, both its dimension and geometry, was computed in [13. This space depends strongly on the curve $\mathcal{C}^{\prime}$ and typically contains many moduli in addition to the translation modulus. Combining this with the discussion above, we see that in heterotic $M$-theory the full moduli space of a small instanton phase transition is $\mathcal{M}^{\prime} \cup \mathcal{M}_{\mathcal{C}^{\prime}}$, where $\mathcal{M}^{\prime}$ is the instanton phase and $\mathcal{M}_{\mathcal{C}^{\prime}}$ the five-brane phase. Generalizing the above discussion, we assume that the complete phase transition can be described by a potential energy function constructed from chiral supermultiplets in some representation $R$ of $G$ and, possibly, in a representation $r$ of an additional gauge symmetry $H$. The origin of field space is an ESP. Away from this point, however, the $H$ symmetry is spontaneously broken and only the moduli parameterizing 
$\mathcal{M}^{\prime} \cup \mathcal{M}_{\mathcal{C}^{\prime}}$ remain massless.

The existence of small instanton phase transitions, from the point of view of holomorphic vector bundles and topology, was demonstrated in 12. In the present paper, by studying the motion of heterotic $M$-theory vacua in moduli space coupled to an expanding Friedmann-Robinson-Walker (FRW) spacetime, we will show that these phase transition can also take place dynamically. Specifically, we will show that an $M$-five-brane in the bulk can dynamically evolve through the ESP, the point in moduli space where the five-brane collides with the end of the $\mathbf{S}^{1} / \mathbf{Z}_{2}$ interval, continuing until the vacuum comes to rest at a local minimum of the non-perturbative superpotential. At this point, which is substantially distant from the ESP, the sheaf over $\mathcal{C}^{\prime}$ has combined with $V^{\prime}$ to form a smooth vector bundle $V$, completing the small instanton phase transition. The dynamical system is trapped at this local minimum by the Hubble friction of the FRW space. We ignore the effect of moduli trapping due to radiation into massless fields at the ESP [15], but will argue that this is insufficient to alter our results. Our results are consistent with those found, in different contexts, in [14, 15, 16, 17, 18. These papers ignore any non-perturbative potentials that might arise and discuss flat moduli space. They find that the moduli are trapped, both by Hubble friction and quantum radiation, exactly at the ESP, which, they go on to argue, gives it a special physical meaning. We note that were we to turn off the non-perturbative superpotential in heterotic $M$-theory, one would arrive at precisely the same conclusion. However, turning on this potential completely alters this result and allows the completion of the small instanton phase transition.

Throughout this paper, for notational and computational simplicity, we will take all fields to be dimensionless and choose units in which $M_{P}=1$. 


\section{Moduli Trapping without a Non-Perturbative Superpotential:}

In this section, we consider the dynamics of small instanton transitions ignoring the non-perturbative superpotential generated by worldsheet instantons. In this case, the moduli space $\mathcal{M}^{\prime} \cup \mathcal{M}_{\mathcal{C}^{\prime}}$ is the flat locus of the potential. As discussed above, the dimension and geometry of this moduli space is completely understood, at least for a wide range of base spaces $B$ and small instanton curves $\mathcal{C}^{\prime}$. However, $\mathcal{M}^{\prime}$ and $\mathcal{M}_{\mathcal{C}^{\prime}}$ are both, generically, multi-dimensional. For example, the small instanton discussed in the introduction had $\mathcal{M}^{\prime}$ parameterized by 12 complex fields. Unfortunately, a detailed calculation of dynamics in a multi-dimensional moduli space is not only unenlightening in its complexity, but is far beyond our computational power. For this reason, we will restrict our discussion to a simplified moduli space, one in which both $\mathcal{M}^{\prime}$ and $\mathcal{M}_{\mathcal{C}^{\prime}}$ are taken to be one-dimensional. We denote the complex modulus of $\mathcal{M}^{\prime}$, the instanton phase, by $\psi$. Physically, this modulus describes the "smoothing out" of the small instanton sheaf into the smooth vector bundle $V$. The complex modulus of $\mathcal{M}_{\mathcal{C}^{\prime}}$, the five-brane phase, is written as $\phi$ and represents the translation modulus of the fivebrane. We want to emphasize that the literature describing transitions in different contexts [14, 15, 16, 17, 18] all employ very similar simplifications.

\section{Without Gravitation}

We begin by considering the dynamics of the moduli $\phi$ and $\psi$ in static flat spacetime; that is, we turn off their interaction with gravity. The simplest $N=1$ supersymmetric self-interactions that have precisely a one-dimension instanton phase and a one-dimension five-brane phase are described by the superpotential

$$
W=\lambda \phi^{2} \psi^{2} .
$$

Recall that both $\phi$ and $\psi$ are taken to be dimensionless and, hence, $\lambda$ has mass dimensions 3 in Planck units. Furthermore, we choose the Planck mass $M_{P}$ to be unity. The potential function describing the small instanton phase transition is then given by

$$
\mathcal{V}=\left|\partial_{\phi} W\right|^{2}+\left|\partial_{\psi} W\right|^{2}=4 \lambda^{2}|\phi|^{2}|\psi|^{2}\left(|\phi|^{2}+|\psi|^{2}\right) .
$$


The instanton phase is the one-dimensional complex submanifold defined by $\phi=0$, whereas the five-brane phase is the one-dimensional space given by $\psi=0$. The dynamical calculation is carried out in the four-dimensional real space parameterized by $\operatorname{Re} \phi, \operatorname{Im} \phi, \operatorname{Re} \psi$ and $\operatorname{Im} \psi$. Without loss of generality, one can take $\lambda=1$. Other values of $\lambda$ lead to the same conclusions. In this paper, we will assume that the Calabi-Yau radius and the interval length are chosen, in units where $M_{P}=1$, to be in the phenomenologically acceptable range

$$
R_{C Y} \sim\left(10^{-3}\right)^{-1}, \quad \pi \rho \sim\left(10^{-4}\right)^{-1} .
$$

This implies that in order to achieve the correct phenomenological values for the Newton and gauge coupling parameters, the dimensionless volume and interval moduli should be stabilized at (or be slowly rolling near) the values of order one. In this paper, we will assume that this is the case. This limits $\phi$ to be less than or of the order of unity. For reasons to become clear in the next subsection, we will choose both $\phi$ and $\psi$ moduli to have values much smaller than one.

Let us compute the dynamics with the following initial conditions. Take the initial moduli to be

$$
\phi_{0}=\left(7.4 \times 10^{-2}\right)+\mathbf{i}\left(-4 \times 10^{-3}\right), \quad \psi_{0}=(0)+\mathbf{i}\left(3 \times 10^{-3}\right)
$$

and their initial velocities as

$$
\dot{\phi}_{0}=\left(-3.9 \times 10^{-7}\right)+\mathbf{i}\left(-5.7 \times 10^{-7}\right), \quad \dot{\psi}_{0}=\left(7.9 \times 10^{-6}\right)+\mathbf{i}\left(-6.2 \times 10^{-6}\right) .
$$

These values represent the following physical situation. First, there is a fivebrane in the bulk space substantially separated from the end of the $\mathbf{S}^{1} / \mathbf{Z}_{2}$ interval with a much smaller displacement in the axionic direction. A similar very small displacement occurs in the vector bundle modulus. The five-brane has a small negative velocity in the Re $\phi$ direction which will cause it to collide with the end of the interval and a similar velocity in the axion direction. Substantially larger velocities are given to both $\operatorname{Re} \psi$ and $\operatorname{Im} \psi$ which enable a possible transition from the five-brane branch to the instanton branch. The equations of motion are solved numerically on MATHEMATICA, with the results for Re $\phi$ and $I m \psi$ presented in Figures 1 and 2. Curve (A) in Figure 1 shows that the five-brane collides with the end of the interval but then continues to oscillate around that point. Similarly, curve (B) shows that the collision initiates a transition in the vector bundle modulus direction 


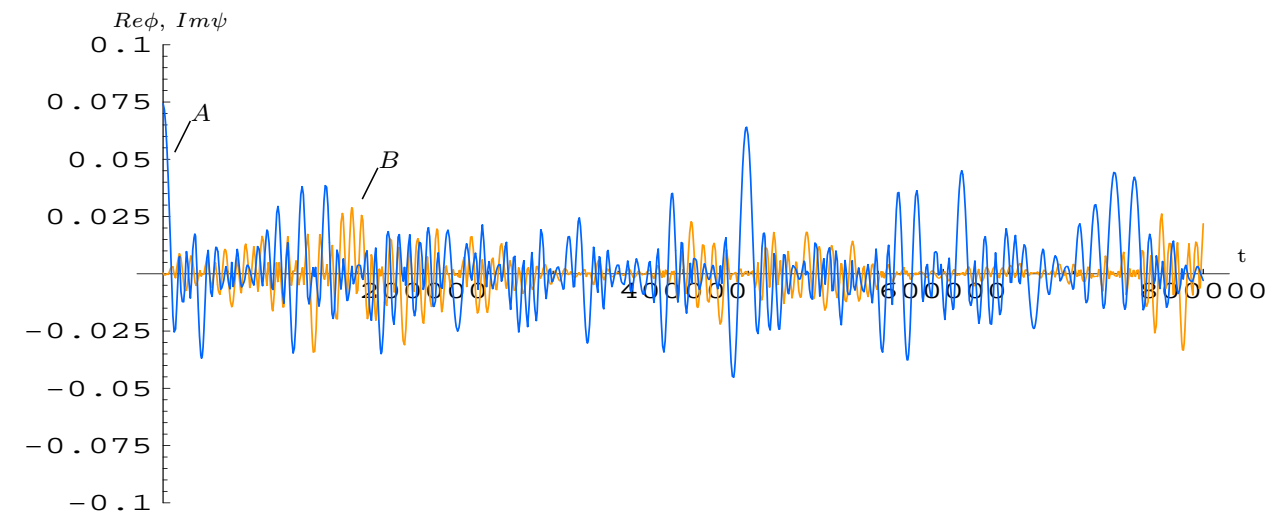

Figure 1: No gravitation and no non-perturbative superpotential: curves (A) and (B) represent the dynamics of $\operatorname{Re} \phi$ and $\operatorname{Im} \psi$ respectively.

which, however, quickly terminates. The resulting motion is an oscillation around the small instanton in the vector bundle direction as well. Figure 2 shows the overall motion in moduli space near the small instanton at the origin of field space. Note that although the amplitude of any individual real modulus increases and decreases quasi-periodically, the overall amplitude remains constant in time, as is clearly indicated in Figure 2. The dynamics of the remaining two real moduli, $\operatorname{Im} \phi$ and $\operatorname{Re} \psi$, is similar, so we will not display them.

These results are easy to explain. First of all, in both the five-brane and instanton branches, the potential energy function has walls which become progressively steeper as one moves away from the origin of field space. Hence, the moving moduli see a "concave" potential that always reflects them back toward the origin. This accounts for the oscillation around the small instanton at the origin of field space. Secondly, the lack of any damping, be it Hubble friction or any other mechanism, means that the overall oscillation amplitude persists. The final conclusion is that the phase transition is never completed. Instead the system oscillates endlessly around the small instanton. Note that these results are consistent with those found in 14, 15, 16, 17, 18, when analyzing undamped motion in different contexts. 


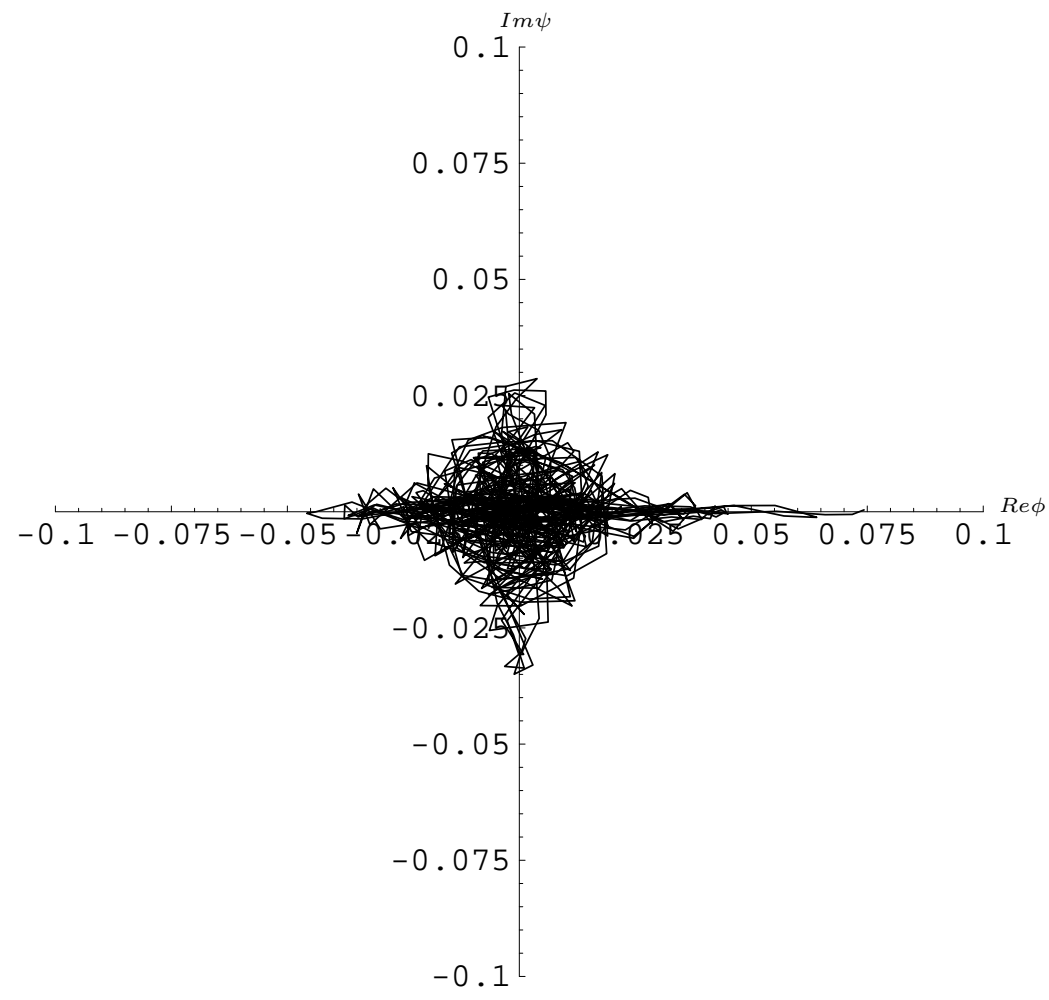

Figure 2: No gravitation and no non-perturbative superpotential: the combined motion of $\operatorname{Re} \phi$ and $\operatorname{Im} \psi$ near the origin of moduli space.

\section{With Gravitation}

Let us now consider the moduli $\phi$ and $\psi$ interacting with gravity. In this case, the dynamics is described by $N=1$ chiral multiplets coupled to supergravity and one must specify both a superpotential $W$ and a Kahler potential $K$. The superpotential remains the same, namely

$$
W=\lambda \phi^{2} \psi^{2} .
$$

In general, the Kahler potential of moduli in heterotic $M$-theory is unknown. However, near the origin of field space, that is, when the magnitudes of the moduli are much smaller than unity, this can be taken to be "flat". Therefore, as long as we restrict $\phi$ and $\psi$ describing our small instanton phase transition to be small, the Kahler potential is given by

$$
K=|\phi|^{2}+|\psi|^{2} .
$$


This is one reason why we chose the initial conditions for $\phi$ and $\psi$ in the previous subsection to be much less than one. The potential function describing the small instanton phase transition is now

$$
\mathcal{V}=e^{K}\left(\left|D_{\phi}\right|^{2}+\left|D_{\psi}\right|^{2}-3|W|^{2}\right),
$$

where $W$ and $K$ are given in (6) and (17) respectively and $D$ is the Kahler covariant derivative. This is a rather complicated function of the moduli. However, for $\phi$ and $\psi$ much smaller than unity, one need only consider the lowest order interactions. All terms that are higher order in $M_{P}^{-1}$ can safely be ignored. The result is that one can analyze the dynamics with the same potential as in the non-gravitational case, that is,

$$
\mathcal{V}=4 \lambda^{2}|\phi|^{2}|\psi|^{2}\left(|\phi|^{2}+|\psi|^{2}\right)
$$

As previously, we will choose $\lambda=1$ and only consider the region of moduli space where $\phi$ and $\psi$ are much smaller than one. Additionally, one must give an ansatz for the spacetime geometry. We will assume a metric of the form

$$
d s^{2}=-d t^{2}+a(t)^{2} \overrightarrow{d x}^{2}
$$

corresponding to a spatially flat manifold of the FRW type. Furthermore, we

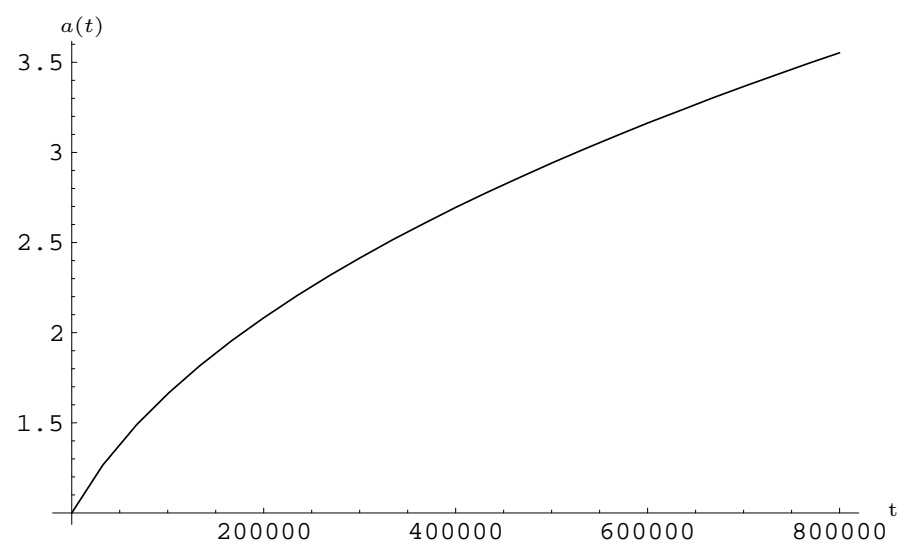

Figure 3: Graph of the FRW expansion parameter $a(t)$.

will only consider metrics that are monotonically expanding. There are, in total, five equations of motion that must be solved simultaneously. To begin 
with, there is a single Einstein equation for the scale parameter $a$. Additionally, there are the four equations of motion for the real and imaginary parts of the moduli $\phi$ and $\psi$. Despite the fact that the potential in (9) is independent of gravitationally induced interactions, non-trivial gravity effects do enter each equation as a Hubble damping term.

Let us compute the dynamics with the following initial conditions. Take the initial value of the scale parameter to be

$$
a_{0}=1 .
$$

It is unnecessary to fix an initial radial velocity. We will continue to choose the initial conditions for the $\phi$ and $\psi$ moduli and their velocities to be those given in (4) and (5) respectively. These values represent exactly the same physical situation as in the previous subsection. Here, however, the moduli evolve with respect to an expanding FRW background spacetime. The equations of motion are solved numerically on MATHEMATICA. The result for the scale parameter $a$ is shown in Figure 3, while those for $\operatorname{Re} \phi$ and $\operatorname{Im} \psi$ are presented in Figures 4 and 5 . Figure 3 shows that $a$ increases monotonically from its initial value, indicating an expanding FRW universe.

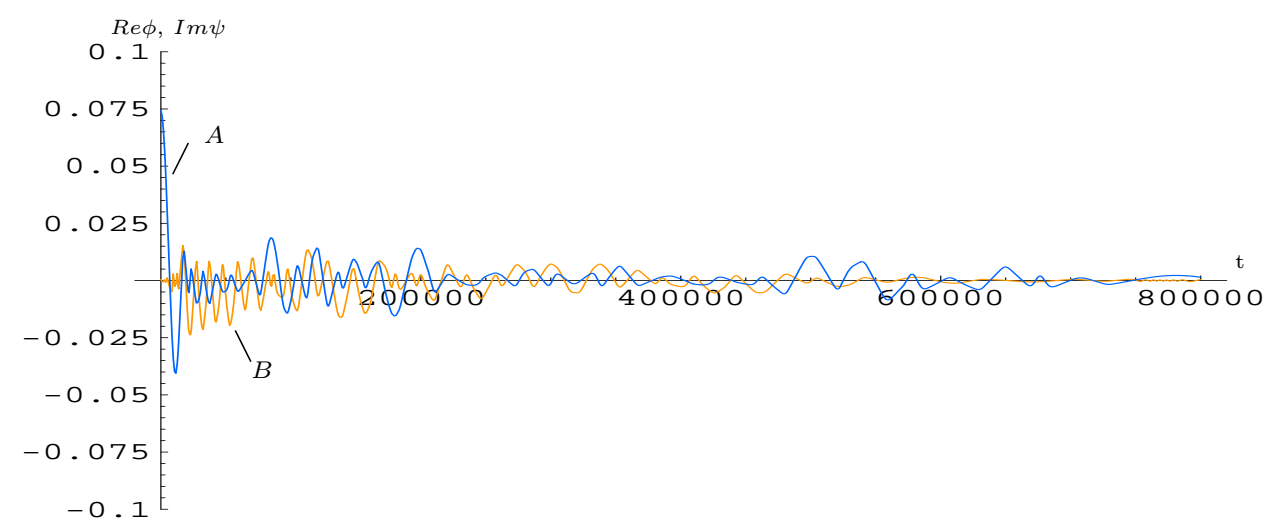

Figure 4: FRW spacetime but no non-perturbative superpotential: curves (A) and (B) represent the dynamics of $\operatorname{Re} \phi$ and $\operatorname{Im} \psi$ respectively.

From curve (A) in Figure 4, one sees that the five-brane collides with the end of the interval and then continues to oscillate around that point. However, the average amplitude and frequency of the oscillation are now strongly damped in time, with Rep converging toward zero. Similarly, curve (B) in 
Figure 4 shows that the collision initiates a transition into the vector bundle modulus direction which then terminates, reverses and begins to oscillates around $\operatorname{Im} \psi=0$. Once again, the average amplitude and frequency of the oscillation in the vector bundle modulus direction are damped in time and $\operatorname{Im} \psi$ converges to zero. Figure 5 shows the overall motion in moduli space

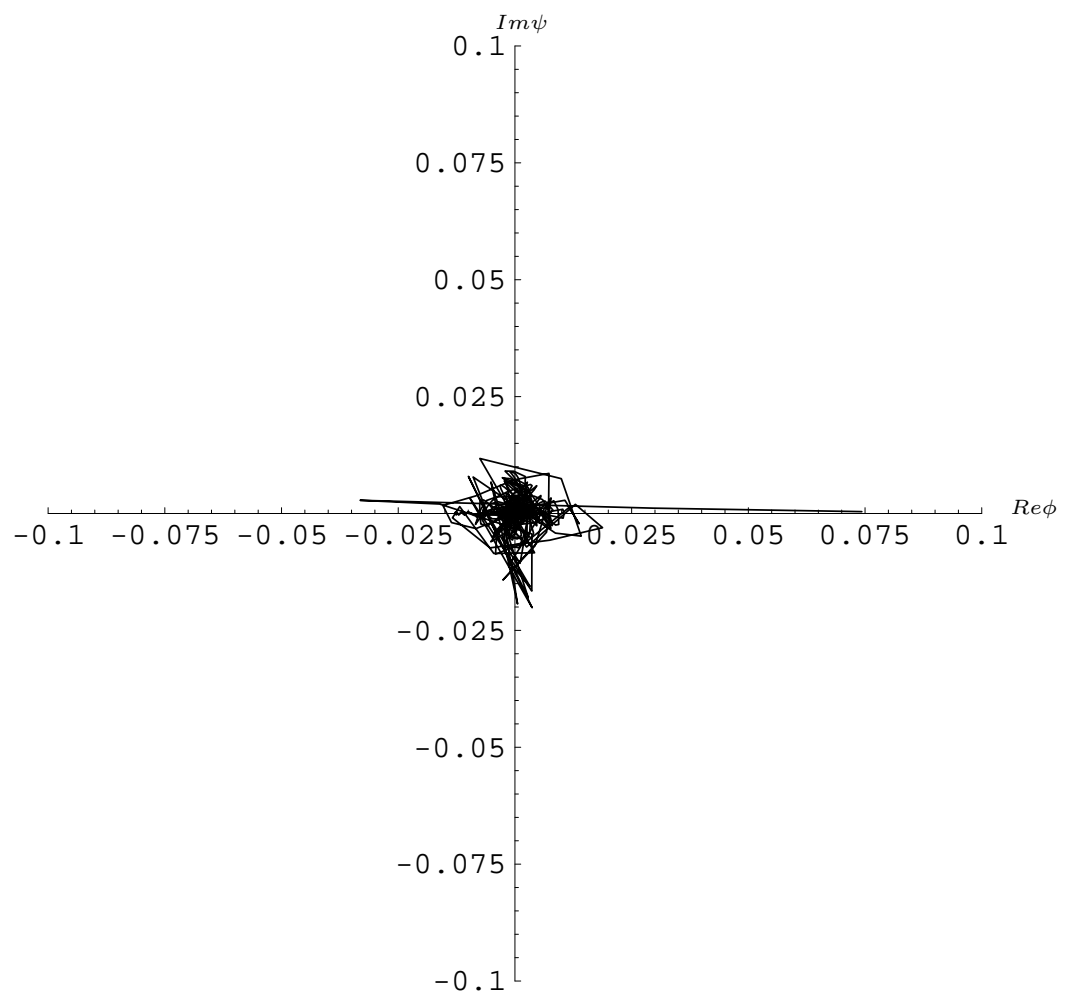

Figure 5: Gravitation but no non-perturbative superpotential: the combined motion of $\operatorname{Re} \phi$ and $\operatorname{Im} \psi$ near the origin of moduli space.

near the small instanton at the origin of field space. Note that the overall amplitude decreases in time. The dynamics of the remaining real moduli,

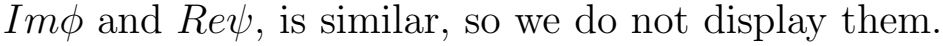

The physical explanation of these results is identical to that given in the previous subsection, with one important exception. The expansion of the universe produces "friction" in the motion of the moduli. This causes their oscillation around the origin of field space to have ever diminishing amplitude and frequency. The physical conclusion is that after collision and a period of 
damped oscillation, the five-brane becomes fixed at the end of the interval. The associated bundle data is the "union" of $V^{\prime}$ and a torsion free sheaf over the curve $\mathcal{C}^{\prime}$. That is, the system approaches the small instanton ESP and the phase transition to a smooth bundle $V$ is never completed. Note that these results are completely consistent with those found in [14, 15, 16, 17, 18] when analyzing damped motion in different contexts.

\section{Moduli Trapping with a Non-Perturbative Superpotential:}

Having established our preliminary results, we now turn to the main discussion in this paper; namely, the dynamics of small instanton transitions in the presence of a non-perturbative superpotential generated by worldsheet instantons. In this case, the moduli space $\mathcal{M}_{\mathcal{C}^{\prime}}$ of the curve remains flat. However, the potential for the vector bundle moduli is now substantially altered, with the result that, generically, the flat moduli space $\mathcal{M}^{\prime}$ is replaced by one or more isolated local minima. To proceed, we must first be more explicit about the form of the non-perturbative interactions.

\section{Heterotic Non-Perturbative Superpotentials}

It was shown in 9] that the superpotential generated by a string wrapped on an isolated curve $\mathcal{C}^{\prime}$ has the form

$$
W_{N P} \propto \operatorname{Pfaff}\left(\psi_{a}\right) e^{-\tau \phi},
$$

where $\psi_{a}$ are a subset of vector bundle moduli and we have assumed, for simplicity, that the moduli space of the five-brane wrapped on $\mathcal{C}^{\prime}$ is onedimensional and parameterized by the dimensionless translation modulus $\phi$. The coefficient $\tau$ is then dimensionless and given by

$$
\tau=\frac{1}{2}(2 \pi)^{1 / 3}\left(\frac{1}{2 \kappa_{11}^{2}}\right)^{1 / 3} \pi \rho v,
$$

where $\rho$ is the size of the $\mathbf{S}^{1} / \mathbf{Z}_{2}$ interval, $v$ is the area of $\mathcal{C}^{\prime}$ and $\kappa_{11}$ is the eleven-dimensional Planck scale. When the Calabi-Yau radius and the interval length are chosen, in units where $M_{P}=1$, to be in the phenomenologically acceptable range eq. (3),$\tau$ is found [19] to be of order 250. Let us recall that 
in our notation the position of the five-brane is dimensionless and bounded by the length of the interval which is of order one.

As mentioned in the introduction, $\operatorname{Pfaff}\left(\psi_{a}\right)$ is the Pfaffian of the Dirac operator twisted by the vector bundle $V$ restricted to the curve $\mathcal{C}^{\prime}$. It is found to be a homogeneous polynomial of the moduli $\psi_{a}$ of the restricted bundle. In general, the Pfaffian is difficult to compute. However, it has been explicitly calculated in [10, 11] for vector bundles with structure group $S U(N)$ restricted to an isolated curve $\mathcal{C}^{\prime}$ in an elliptic Calabi-Yau threefold with base $B=\mathbf{F}_{r}$. For example, choosing $r=1$, a specific $S U(3)$ vector

bundle and $\mathcal{C}^{\prime}=\mathcal{S}$, where $\mathcal{S}$ is an isolated curve in $\mathbf{F}_{1}$, it was shown that the Pfaffian is a homogeneous polynomial of degree 5 in 7 out of the 12 moduli of $\mathcal{M}^{\prime}$. An important pattern that emerges from the results in [10, 11] is that the smaller the dimension of $\mathcal{M}^{\prime}$, the smaller the degree of the homogeneous polynomial. A final comment is that the coefficient of proportionality has not been explicitly computed.

Now consider the simplified moduli space in which both $\mathcal{M}^{\prime}$ and $\mathcal{M}_{\mathcal{C}^{\prime}}$ are one-dimensional and parameterized by the moduli $\psi$ and $\phi$ respectively. In this case, the non-perturbative superpotential $W_{N P}$ in (12) takes the form

$$
W_{N P}=A \psi^{p} e^{-\tau \phi} \text {, }
$$

where $p$ is a small integer and $A$ is a coefficient of mass dimension $3-p$ in Planck units. We now proceed to discuss the moduli dynamics in the presence of superpotential (14).

\section{Without Gravitation}

Again consider the dynamics of the moduli $\phi$ and $\psi$ in static flat spacetime. However, the complete superpotential is now given by

$$
W=\lambda \phi^{2} \psi^{2}+A \psi^{p} e^{-\tau \phi} .
$$

The potential function describing the small instanton phase transition is

$$
\mathcal{V}=\left|\partial_{\phi} W\right|^{2}+\left|\partial_{\psi} W\right|^{2}
$$

where

$$
\partial_{\phi} W=2 \lambda \phi \psi^{2}-\tau A \psi^{p} e^{-\tau \phi}, \quad \partial_{\psi} W=2 \lambda \phi^{2} \psi+p A \psi^{p-1} e^{-\tau \phi} .
$$


The minimum of this potential has two distinct types of separated loci. The first, specified by

$$
\psi=0, \quad \phi \quad \text { arbitrary, }
$$

is the moduli space $\mathcal{M}_{\mathcal{C}^{\prime}}$ of $\mathcal{C}^{\prime}$. However, the moduli space $\mathcal{M}^{\prime}$ is now replaced by $p-2$ isolated points located at

$$
\psi=\left(-\frac{2 \lambda p}{\tau^{2} A e^{p}}\right)^{1 /(p-2)} e^{\frac{2 \pi i n}{p-2}}, \quad \operatorname{Re} \phi=-\frac{4}{\tau}, \quad \operatorname{Im} \phi=0,
$$

where $n=1,2, \ldots, p-2$.

To carry out an explicit calculation of the moduli dynamics, one must specify the values of the parameters in superpotential (15). Without loss of generality, choose $\lambda=1$. For specificity, we will take

$$
\tau=400, \quad p=4, \quad A=\frac{1}{200 e^{4}} .
$$

The choice for $\tau$ is consistent with the phenomenologically acceptable values of $R_{C Y}$ and $\pi \rho$ given in (3). Similarly, in view of the discussion above, $p=4$ is a reasonable value for the degree of the Pfaffian polynomial. The choice of $A$ in (20) is less well-motivated. It is taken because it enhances the distance between the local minimum and the ESP in moduli space, as can be seen from (19). For the parameters in (20), the local minima in (19) become

$$
\operatorname{Re} \psi=0, \quad \operatorname{Im} \psi= \pm 0.1, \quad \operatorname{Re} \phi=-0.01, \quad \operatorname{Im} \phi=0 .
$$

Similar physical conclusions will be reached for a wide range of all three parameters.

With these choices, we compute the moduli dynamics using initial conditions similar to those given in (4) and (5). The physical situation is identical to that described in the previous section. The moduli equations of motion are solved numerically on MATHEMATICA, with the results for Re $\phi$ and $\operatorname{Im} \psi$ shown in Figure 6.

Figure $6(C)$ indicates that the five-brane collides with the end of the interval but then, eventually, continues to oscillate without damping around that point. Now, however, there is a new phenomenon. Note that immediately after the collision the Re $\phi$ modulus gets "hung up" at the negative value corresponding to the local minima in (21). It lingers there for a considerable time, before rolling back through the origin and continuing its oscillation 


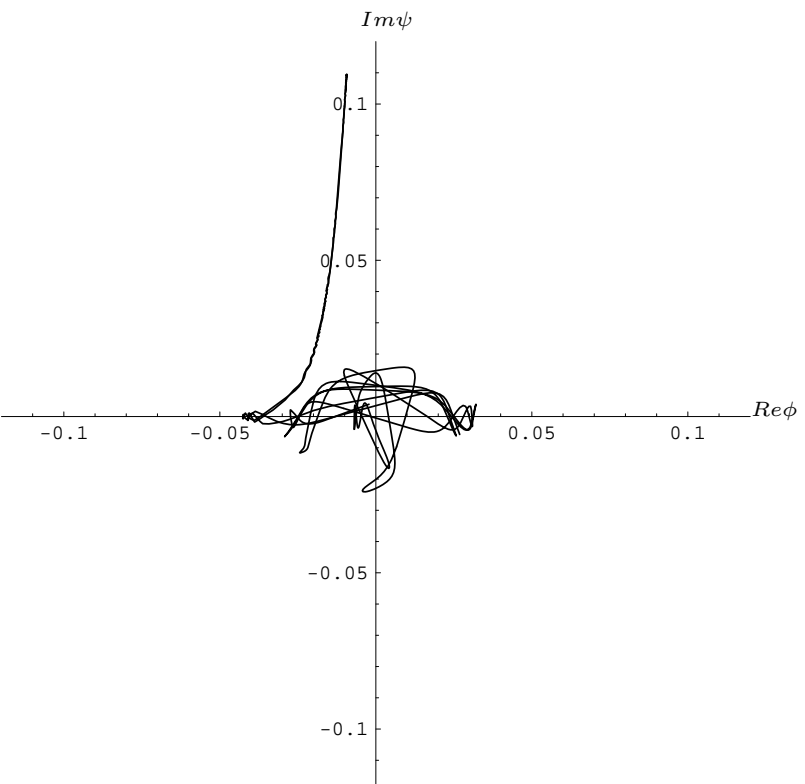

$(A)$

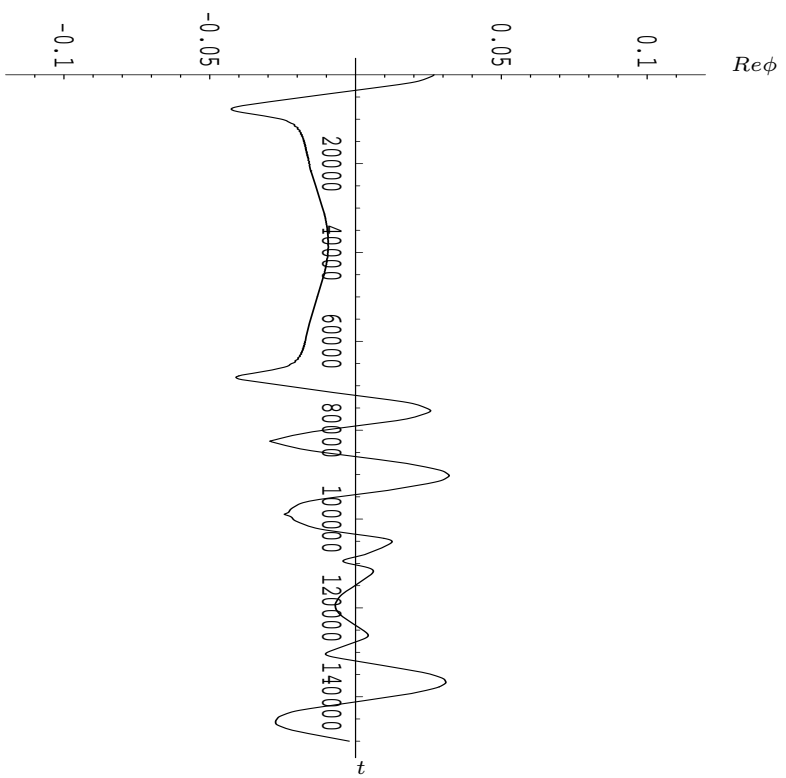

$(C)$

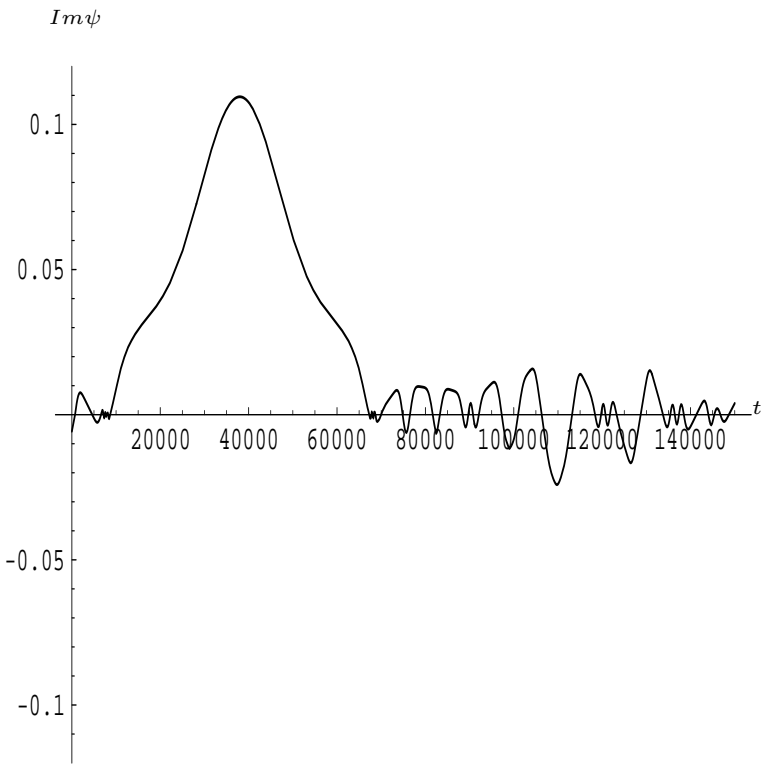

$(B)$

Figure 6: (C) and (B) represent the dynamics of Re $\phi$ and $\operatorname{Im} \psi$ respectively for the case of a non-perturbative superpotential but no gravity. (A) gives the combined motion of these moduli. 
around the small instanton point. Similar, but more dramatic, behaviour for $\operatorname{Im} \psi$ is shown in Figure 6(B). After a few small oscillations around the origin of field space, the $\operatorname{Im} \psi$ modulus rolls all the way out to the value +0.1 corresponding to one of the local minima in (21). It stays in the vicinity of this minimum for some time, before rolling back through the origin and beginning an undamped oscillation around the small instanton. The choice of +0.1 rather than the other minimum at -0.1 is due to the initial conditions. These can be changed to select the other local minimum. The behaviour of both moduli is graphically presented in Figure 6(A), where the excursion of the moduli to the local minimum and back is clearly visible. The other two real moduli, $\operatorname{Im} \phi$ and $R e \psi$, simply oscillate with undamped motion around the origin of field space, so we will not display them.

Comparing these results with the static flat case in Section 2, we conclude that the non-perturbative superpotential has a dramatic effect on the dynamics of the moduli. Specifically, the moduli now evolve far from the origin of field space, seeking, and reaching, an isolated local minimum of the potential. However, since there is no mechanism for damping, the moduli roll through the isolated minimum, returning to oscillate around the small instanton. Were one to wait for a sufficiently long time, the moduli might once again briefly return to one of the two local minima, only to roll out again and oscillate around the origin of field space. The lack of any damping means that the overall oscillation amplitude persists. We conclude that, despite the existence of isolated local minima, the phase transition is never completed.

\section{With Gravitation}

Now let the moduli $\phi$ and $\psi$ interact with gravity. The superpotential remains the same, namely

$$
W=\lambda \phi^{2} \psi^{2}+A \psi^{p} e^{-\tau \phi} .
$$

Since the values of all moduli we consider, including the isolated local minima, are much smaller than unity, one can continue to choose the Kahler potential to be

$$
K=|\phi|^{2}+|\psi|^{2}
$$

Furthermore, since $\phi$ and $\psi$ are much smaller than one, the complicated potential function in (8) simplifies to

$$
\mathcal{V}=\left|\partial_{\phi} W\right|^{2}+\left|\partial_{\psi} W\right|^{2}
$$


That is, all terms that are higher order in $M_{P}^{-1}$ can be ignored. The potential function is then identical to the non-gravity case with identical minima; namely, the five-brane branch (18) and the $p-2$ isolated minima given in (19). As in the previous subsection, we choose $\lambda=1$ and take

$$
\tau=400, \quad p=4, \quad A=\frac{1}{200 e^{4}} .
$$

For these parameters, the two isolated minima in (19) are again

$$
\operatorname{Re} \psi=0, \quad \operatorname{Im} \psi= \pm 0.1, \quad \operatorname{Re} \phi=-0.01, \quad \operatorname{Im} \phi=0 .
$$

Additionally, one must give an ansatz for the spacetime geometry. We will continue to assume an expanding FRW metric of the form given in (10).

Let us now compute the dynamics of the moduli $\phi$ and $\psi$, as well as the spacetime expansion parameter $a$, using the same initial conditions as in Section 2, namely, (4), (5) and (11). The physical situation remains the same as previously. We use MATHEMATICA to numerically solve the equations of motion. The result for the scale parameter $a$ is similar to that shown in Figure 3 and, hence, we won't present it here. The dynamics of Re $\phi$ and $\operatorname{Im} \psi$ is given in Figure 7.

Figure $7(C)$ indicates that the five-brane collides with the end of the interval. Immediately after the collision, the Re $\phi$ modulus gets "hung up" at the negative value corresponding to the local minima in (26). Now, however, the modulus is "trapped" near this value and does not return to the origin of field space. Rather, it oscillates with ever decreasing amplitude and frequency around $\operatorname{Re} \phi=-0.01$, eventually coming to rest at this point. The behaviour of $I m \psi$ is similar. Figure $7(B)$ shows that after a few small oscillations around the origin of field space, the $\operatorname{Im} \psi$ modulus rolls all the way out to the value +0.1 corresponding to one of the local minima of (26). Again, this local minimum, rather than -0.1 , is selected by the initial conditions. However, unlike in the previous subsection, the modulus is now "trapped" near this minimum, oscillating around it with ever decreasing amplitude and frequency. It eventually comes to rest at $\operatorname{Im} \psi=+0.1$. The behaviour of both moduli is graphically presented in Figure $7(A)$. Here, the excursion of the moduli to the local minimum, and the phenomenon of their being trapped at that point, is clearly visible. The other two real moduli, $\operatorname{Im} \phi$ and $R e \psi$, simply oscillate with decreasing amplitude and frequency around the origin of field space. Hence, we will not display them. 


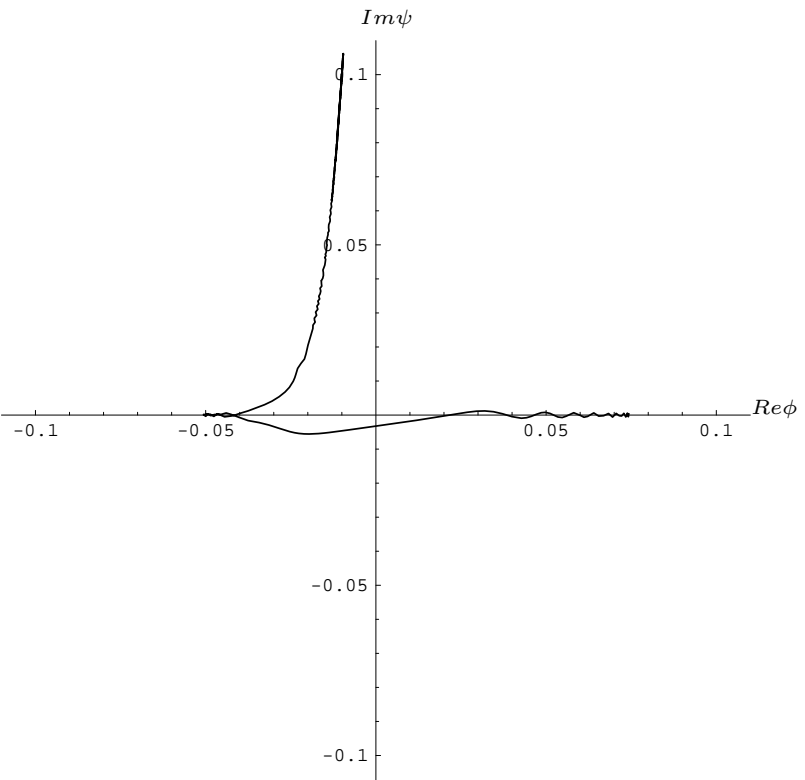

$(A)$

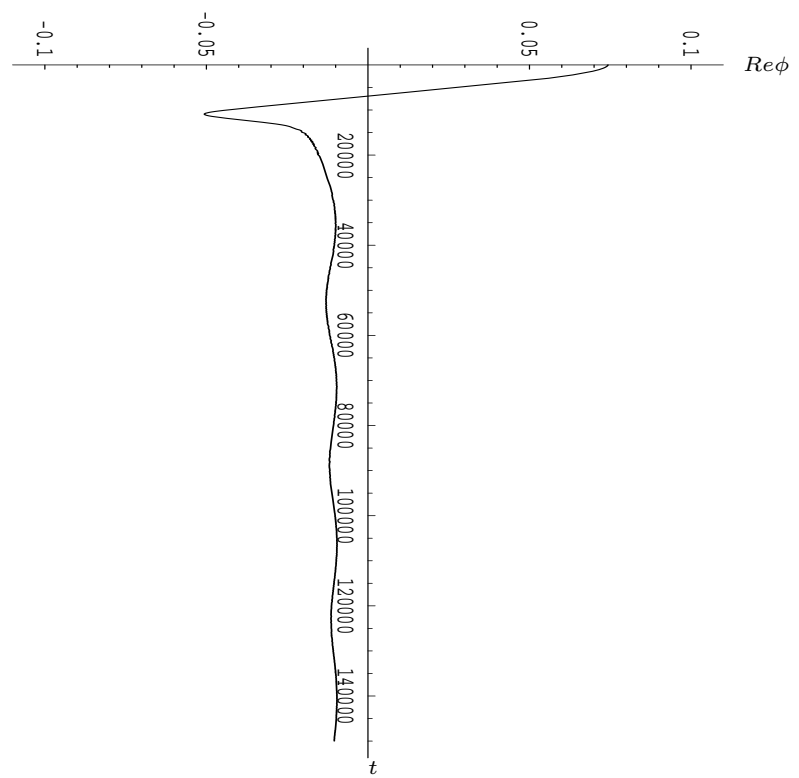

$(C)$

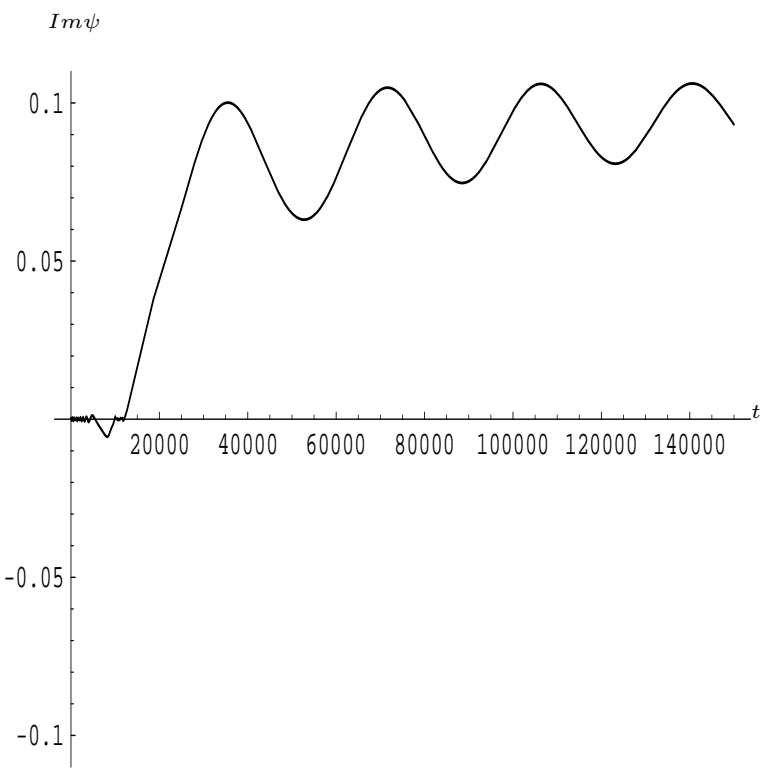

$(B)$

Figure 7: (C) and (B) represent the dynamics of $\operatorname{Re} \phi$ and $\operatorname{Im} \psi$ respectively for a nonperturbative superpotential in an expanding FRW spacetime. (A) gives the combined motion of these moduli. 
Comparing these results with those in the previous subsection, we see that the effect of the expanding FRW geometry on the dynamics of the $\phi$ and $\psi$ moduli is dramatic. Previously, without gravitation, these moduli evolved out to the local minimum of the non-perturbative superpotential. However, because of the absence of any mechanism for damping, the moduli eventually rolled out of the local minimum and back to the origin of field space. They oscillated forever with no loss of amplitude or frequency around the small instanton, occasionally, perhaps, temporarily revisiting one of the two local minima. When coupled to the expanding FRW spacetime, however, this behaviour is completely modified. The results in Figure7 graphically confirm that the moduli now evolve out to the local minimum and are trapped there by gravitational damping. The physical reason is clear. Immediately after collision, the moduli have sufficient "energy" to overcome a potential barrier and roll into a valley surrounding the local minimum. However, the energy is rapidly "red-shifted" by the expanding geometry. The result is that the moduli can no longer cross the potential barrier to return to the origin of field space. The continued loss of energy to gravitational expansion causes the moduli to oscillate with smaller and smaller amplitude and frequency around the minimum, eventually coming to rest there. That is, they are gravitationally trapped at the isolated minimum.

Before drawing our final conclusions, we would like to comment on the effect of radiative damping on the moduli dynamics. As discussed in [15], radiative damping occurs at an ESP, where the number of massless states and, hence, the quantum mechanical amplitude to produce light particles is greatly enhanced. This leads to radiative "friction" on the moduli dynamics, damping their motion. Thus far in this paper we have ignored this effect, considering only gravitational damping. Could the inclusion of radiative damping change our conclusions, perhaps trapping the moduli at the ESP? The answer is no. The results reported in [15] show that although radiative damping is a strong effect, it requires the moduli to oscillate several times around the ESP before their amplitude is substantially reduced. However, as is clear from Figures $7(C)$ and $7(B)$, the transition of $\phi$ and $\psi$ from the vicinity of the ESP to that of the isolated minimum occurs immediately upon collision, within less than one oscillation (Note that the small amplitude oscillations of $\operatorname{Im} \psi$ in Figure $7(B)$ all occur prior to the collision and not near the ESP). Furthermore, they never return to the ESP. It is safe to conclude, therefore, that radiative damping at the ESP simply has no opportunity to effect the moduli dynamics and can be safely ignored for the process 
under discussion in this paper. In fact, radiative damping helps enable our transition to the isolated minimum. The reason is that there is also an enhanced number of light states at the local minimum. Once trapped in its vicinity, radiative damping will work with gravitational damping to quickly bring the moduli to rest at the isolated minimum.

To conclude: In an expanding FRW spacetime, the collision of a five-brane with an end of the $\mathbf{S}^{1} / \mathbf{Z}_{2}$ interval passes through the small instanton point, described by the union of a vector bundle $V^{\prime}$ and a torsion free sheaf over the five-brane curve, and then continues to an isolated minimum of the nonperturbative superpotential where the vector bundle is smooth. Gravitational (and radiative) damping traps the moduli at the isolated minimum, thus completing the small instanton phase transition to the smooth vector bundle $V$. The moduli are not attracted to the ESP in this process.

\section{Discussion:}

It has been demonstrated in this paper that not only is a small instanton phase transition mathematically consistent, as shown in [12], but it can also be realized dynamically. The results were presented for a specific choice of parameters and initial conditions. However, we have explored the dynamics of phase transitions for a range of parameters and initial conditions and found that the transition is completed for many different values. However, this is not always the case. Some choices do lead to trapping at the ESP, presumably because the energy is either too small to roll into the valley of an isolated minimum or so large that it rolls out again. Be this as it may, initial conditions leading to a completed phase transition are ubiquitous and demonstrate that a nonperturbative addition to the potential can dramatically alter the results of [14, 15, 16, 17, 18.

\section{Acknowledgments:}

We are grateful to Volker Braun for discussions of the intricacies of MATHEMATICA. The work of Evgeny Buchbinder is supported by NSF grant PHY0070928. The research of Alexander Borisov and Burt Ovrut is supported in part by the Department of Physics and the Math/Physics Research Group at the University of Pennsylvania under cooperative research agreement DE- 
FG02-95ER40893 with the U. S. Department of Energy and an NSF Focused Research Grant DMS0139799 for "The Geometry of Superstrings."

\section{References}

[1] E. Witten, "Small Instantons in String Theory," Nucl.Phys. B460 (1996) 541-559 hep-th/9511030.

[2] P. Horava and E. Witten, "Heterotic and Type I String Dynamics from Eleven Dimensions," Nucl.Phys. B460 (1996) 525-540 hep-th/9510209; P. Horava and E. Witten, "Eleven-Dimensional Supergravity on a Manifold with Boundary," Nucl.Phys. B475 (1996) 94-114 hep-th/9603142.

[3] A. Lukas, B. A. Ovrut, and D. Waldram, "On the Four-Dimensional Effective Action of Strongly Coupled Heterotic StringTheory", Nucl.Phys. B532 (1998) 43-82 hep-th/9710208; A. Lukas, B. A. Ovrut, K. S. Stelle, and D. Waldram, "The Universe as a Domain Wall", Phys.Rev. D59 (1999) 086001 hep-th/9803235; A. Lukas, B. A. Ovrut, K. S. Stelle, and D. Waldram, "Heterotic M-theory in Five Dimensions", Nucl.Phys. B552 (1999) 246-290 hep-th/9806051.

[4] R. Friedman, J. Morgan and E. Witten, "Vector Bundles And F Theory," Commun.Math.Phys. 187 (1997) 679-743 hep-th/9701162; R. Donagi, "Principal Bundles on Elliptic Fibrations," Asian J. Math 1 (1997) 214-223 alg-geom/9702002.

[5] R. Donagi, A. Lukas, B. A. Ovrut and D. Waldram, "Non-Perturbative Vacua and Particle Physics in M-Theory," JHEP 9905 (1999) 018 hep-th/9811168; R. Donagi, A. Lukas, B. A. Ovrut and D. Waldram, "Holomorphic Vector Bundles and Non-Perturbative Vacua in M-Theory," JHEP 9906 (1999) 034 hep-th/9901009; R. Donagi, J. Khoury, B. A. Ovrut, P. J. Steinhardt and N. Turok, "Visible Branes with Negative Tension in Heterotic M-Theory," JHEP 0111 (2001) 041 hep-th/0105199; R. Donagi, B. A.Ovrut, T. Pantev and R. Reinbacher, "SU(4) Instantons on Calabi-Yau Threefolds with $Z_{2} \times Z_{2}$ Fundamental Group," JHEP 0401 (2004) 02 hep-th/0307273; Y.-H. He, B. A. Ovrut, R. Reinbacher, "The Moduli of Reducible Vector Bundles," JHEP 0403 (2004) 043 hep-th/0306121. 
[6] E. I. Buchbinder, R. Donagi and B. A. Ovrut, "Vector Bundle Moduli and Small Instanton Transitions," JHEP 0206 (2002) 054 hep-th/0202084; E. I. Buchbinder, B. A. Ovrut and R. Reinbacher, "Instanton Moduli in String Theory," JHEP 0504 (2005) 008 hep-th/0410200.

[7] K. Becker, M. Becker and A. Strominger, "Fivebranes, Membranes and Non-Perturbative String Theory," Nucl.Phys. B456 (1995) 130152 hep-th/9507158; E. Witten, "Non-Perturbative Superpotentials In String Theory," Nucl.Phys. B474 (1996) 343-360 hep-th/9604030; J. Harvey and G. Moore, "Superpotentials and Membrane Instantons," hep-th/9907026.

[8] G. Moore, G. Peradze and N. Saulina, "Instabilities in heterotic Mtheory induced by open membrane instantons," Nucl.Phys. B607 (2001) 117-154 hep-th/0012104; E. Lima, B. A. Ovrut, J. Park and R. Reinbacher, "Non-Perturbative Superpotentials from Membrane Instantons in Heterotic M-Theory," Nucl.Phys. B614 (2001) 117-170 hep-th/0101049|; E. Lima, B. A. Ovrut and J. Park, "Five-Brane Superpotentials in Heterotic M-Theory," Nucl.Phys. B626 (2002) 113-164 hep-th/0102046.

[9] E. Witten, "World-Sheet Corrections Via D-Instantons," JHEP 0002 (2000) 030 hep-th/9907041.

[10] E. I. Buchbinder, R. Donagi and B. A. Ovrut, " Superpotentials for Vector Bundle Moduli," Nucl.Phys. B653 (2003) 400-420 hep-th/0205190.

[11] E. I. Buchbinder, R. Donagi and B. A. Ovrut, "Vector Bundle Moduli Superpotentials in Heterotic Superstrings and M-Theory," JHEP 0207 (2002) 066 hep-th/0206203.

[12] B. A. Ovrut, T. Pantev and J. Park, "Small Instanton Transitions in Heterotic M-Theory," JHEP 0005 (2000) 045 hep-th/0001133.

[13] R. Donagi, B. A. Ovrut and D. Waldram, "Moduli Spaces of Fivebranes on Elliptic Calabi-Yau Threefolds," JHEP 9911 (1999) 030 hep-th/9904054.

[14] M.Brandle and A. Lukas, "Flop Transitions in M-Theory Cosmology," Phys.Rev.D68 024030, 2003 hep-th/0212263. 
[15] L. Kofman, A. Linde, X. Liu, A. Maloney, L. McAllister and E. Silverstein, "Beauty is Attractive: Moduli Trapping at Enhanced Symmetry Points," JHEP 0405 (2004) 030 hep-th/0403001.

[16] T. Mohaupt and F. Saueressig, "Dynamical Conifold Transitions and Moduli Trapping in M-Theory Cosmology," JCAP 0501 (2005) 006 hep-th/0410273.

[17] A. Lukas, E. Palti, P. M. Saffin, "Type IIB Conifold Transitions in Cosmology," Phys.Rev.D71 066001, 2005 hep-th/0411033

[18] S. A. Abel and J. Gray, "On the chaos of D-brane phase transitions," hep-th/0504170.

[19] E. I. Buchbinder and B. A. Ovrut, "Vacuum Stability in Heterotic MTheory," Phys.Rev. D69 (2004) 086010 hep-th/0310112. 